\title{
Correction to: The uncertainty with using risk prediction models for individual decision making: an exemplar cohort study examining the prediction of cardiovascular disease in English primary care
}

\author{
Alexander Pate ${ }^{1 *}$, Richard Emsley ${ }^{2}$, Darren M. Ashcroft ${ }^{3,4}$, Benjamin Brown ${ }^{4,5}$ and Tjeerd van Staa ${ }^{1,6}$
}

Correction to: BMC Med 17:134

https://doi.org/10.1186/s12916-019-1368-8

The original article [1] contained an error in the abstract. The mentioned cohort size now correctly states ' $N=3,855,660$ '.

\begin{abstract}
Author details
${ }^{1}$ Centre of Health eResearch, School of Health Sciences, Faculty of Biology, Medicine and Health, The University of Manchester, Oxford Road, Manchester M13 9PL, UK. ${ }^{2}$ Department of Biostatistics and Health Informatics, Institute of Psychiatry, Psychology and Neuroscience, King's College London, De

Crispigny Park, London SE5 8AF, UK. ${ }^{3}$ NIHR Greater Manchester Patient Safety Translational Research Centre, School of Health Sciences, Faculty of Biology, Medicine and Health, The University of Manchester, Oxford Road, Manchester M13 9PL, UK. ${ }^{4}$ NIHR School for Primary Care Research, Centre for Primary Care, Division of Population of Health, Health Services Research and Primary Care, Manchester Academic Health Science Centre, University of Manchester, Manchester M13 9PL, UK. ${ }^{5}$ Public Health England North West, 3 Piccadilly Place, London Road, Manchester M1 3BN, UK. ${ }^{6}$ Division ofPharmacoepidemiology and Clinical Pharmacology, Utrecht Institute of Pharmaceutical Sciences, Utrecht University, Utrecht, Netherlands.
\end{abstract}

Published online: 10 August 2019

\section{Reference}

1. Pate A, Emsley R, Ashcroft DM, Brown B, van Staa T. The uncertainty with using risk prediction models for individual decision making: an exemplar cohort study examining the prediction of cardiovascular disease in English primary care. BMC Med. 2019;17:134 https:/doi.org/10.1186/s12916-019-1368-8.

\footnotetext{
* Correspondence: alexander.pate@manchester.ac.uk

${ }^{1}$ Centre of Health eResearch, School of Health Sciences, Faculty of Biology, Medicine and Health, The University of Manchester, Oxford Road, Manchester M13 9PL, UK

Full list of author information is available at the end of the article
}

(c) The Author(s). 2019 Open Access This article is distributed under the terms of the Creative Commons Attribution 4.0 International License (http://creativecommons.org/licenses/by/4.0/), which permits unrestricted use, distribution, and reproduction in any medium, provided you give appropriate credit to the original author(s) and the source, provide a link to the Creative Commons license, and indicate if changes were made. The Creative Commons Public Domain Dedication waiver (http://creativecommons.org/publicdomain/zero/1.0/) applies to the data made available in this article, unless otherwise stated. 